Article

\title{
Economic Risk and Efficiency Assessment of Fisheries in Abu-Dhabi, United Arab Emirates (UAE): A Stochastic Approach
}

\author{
Eihab Fathelrahman ${ }^{1, *}$, Aydin Basarir ${ }^{1, \dagger}$, Mohamed Gheblawi ${ }^{1, \dagger}$, Sherin Sherif ${ }^{2, \dagger}$ \\ and James Ascough, II ${ }^{3, \dagger}$
}

1 Department of Agribusiness and Consumer Sciences, College of Food and Agriculture, United Arab Emirates University, P.O. Box 15551, Al Ain, United Arab Emirates;

E-Mails: abasarir@uaeu.ac.ae (A.B.); gheblawi@uaeu.ac.ae (M.G.)

2 Economics and Agribusiness Department, College of Agriculture, Alexandria University, El-Shatby, Alexandria, Egypt; E-Mail: sherifsherin@yahoo.com

3 United Sates Department of Agriculture (USDA), Agricultural Research Services (ARS), Northern Plain Area (NPA), Agricultural Systems Research Unit (ASRU), 2150 Centre Avenue, Building D, Suite 200, Fort Collins, CO 80526, USA; E-Mail: jim.ascough@ars.usda.gov

$\dagger$ These authors contributed equally to this work.

* Author to whom correspondence should be addressed; E-Mail: eihab.fathelrahman@uaeu.ac.ae; Tel.: +971-3-713-4589; Fax: +971-3-713-6903.

Received: 28 February 2014; in revised form: 4 June 2014 / Accepted: 5 June 2014 /

Published: 17 June 2014

\begin{abstract}
The fishing industry in Abu-Dhabi, United Arab Emirates (UAE), plays an important role in diversifying food sources in order to enhance national food security. The fishing industry is facing an increasing risk that may impact the sustainability (i.e., quantity and quality) of the fish caught and consumed in the UAE. Therefore, the main objective of this study is to analyze common Abu-Dhabi fishing management alternatives using various stochastic dominance techniques (i.e., first/second degree stochastic dominance, stochastic dominance with respect to a function and stochastic efficiency with respect to a function) to assess the risk facing UAE fishermen. The techniques represent a risk assessment continuum, which can provide a ranking of management alternatives to improve decision making outcomes and help maintain long-term UAE fishing sustainability. Data for the stochastic dominance analyses were obtained from a cross-sectional survey conducted through face-to-face interviews of Abu Dhabi, UAE, fishermen. Analysis of fishing methods, trap sizes and trap numbers using stochastic efficiency with respect to a function (SERF) showed that fishermen efficient practices were not the same for risk-neutral
\end{abstract}


fishermen compared to risk averse fishermen. Overall, the stochastic dominance results illustrated the importance of considering both attitude towards risk and economic inefficiencies in managing UAE fishery practices and designing successful fishery policies, as well as improving decision-making at the fishermen level.

Keywords: fisheries; technical efficiency; allocative efficiency; stochastic dominance; stochastic efficiency with respect to a function (SERF); socio-economics

\section{Introduction}

Fishing is one of the oldest traditional industries in the United Arab Emirates (UAE) and is typically inherited from former generations. Pearl extraction and fishing were dominant sources of income before oil and natural gas discoveries in the country. The majority of the fish catch originates from the Emirate of Abu Dhabi, which includes more than $65 \%$ of the UAE sea area in its borders. The Food and Agriculture Organization (FAO) [1] reported that UAE fishery policy objectives can be summarized as follows: (1) maintain traditional fishing and produce fresh fish for local markets to serve national food security policy goals; (2) control and support research for the sustainable use of fishery stocks; (3) prohibit destructive fishing practices to protect the marine environment; (4) enhance local fish populations, build artificial reefs and re-stock important local species; and (5) subsidize local fish producers. These policy objectives were translated to Fishery Law No. 23, which was enacted in 1999 to regulate fishery operations in the UAE [1,2].

In order to control fishing efforts, the Emirate of Abu Dhabi introduced new regulations in 2003 [2]. The Emirate has the right to restrict fishing in certain fishing areas, seasons of the year and certain fishing methods, such as traps, nets and threads. Traps in the UAE might consist of a frame of thick steel wire in different shapes, such as triangular cages, cones or igloo-shaped traps. Traps are locally called "Gargor". Nets are made of acrylic fiber, forming different sizes and shapes of grids. Threads include different types of lines and hooks. The Emirate of Abu Dhabi introduced a restriction policy on the number of fish traps per vessel and issued new commercial fishing licenses in 2003. Furthermore, the Emirate required all fish boats to carry a government-issued tag, and at least one UAE national person has to be physically present on vessels during fishing operations.

In order to estimate UAE fishing self-sufficiency ratios, time series for fish production, export, import and consumption have been analyzed. The self-sufficiency ratio (SSR) is a percentage of the locally-produced quantity of product over the total supply available for the consumption of such a product. The total quantity of supply available for consumption is calculated by subtracting product exports from the total of locally-produced product and adding product imports from abroad. The fish catch in UAE increased steadily from 70,000 metric tons in 1982 to 115,000 metric tons in 1999. Production then started declining until it reached 77,700 metric tons in 2010. Meanwhile, fish consumption in the UAE steadily increased from 76,400 metric tons in 1982 to 192,640 metric tons in 2010. The differences between consumption and production are filled with imports from other countries to balance the food production in addition to imports (supply) and consumption (demand) in the UAE. Fish imports in the UAE increased from 8780 metric tons in 1982 to 132,720 metric tons in 
2010. The UAE fish self-sufficiency ratio (SSR) historically increased from $92 \%$ in 1982 to $129 \%$ in 1991, when the country was exporting fish to neighboring countries. However, the SSR has declined in recent years (40\% in 2010) to the lowest level since 1982 (Figure 1), due to several external and internal factors [3]. Factors external to the fishing industry include changes in federal and Abu-Dhabi Emirate regulations in relation to fishing capacity and seasons, increased water pollution in over 40 ports and sites where the fishing industry has expanded in the last three decades (especially after the first Gulf War in 1991 and the invasion of Iraq in 2003), and the reduction or disappearance of favored commercial fish species, such as Hamour, Kan'ad-Chabbat, Sultan Ibrahim and Safi Arabi. Factors internal to the fishing industry include resource management to meet federal and UAE regulations, nationalization and the optimal mix of fishing equipment, boat horsepower and manpower to maximize profit [4]. Figure 2 shows the total fish catch originating from the Emirate of Abu-Dhabi during the period 2001-2010.

Figure 1. UAE fish self-sufficiency ratio (1982-2010).

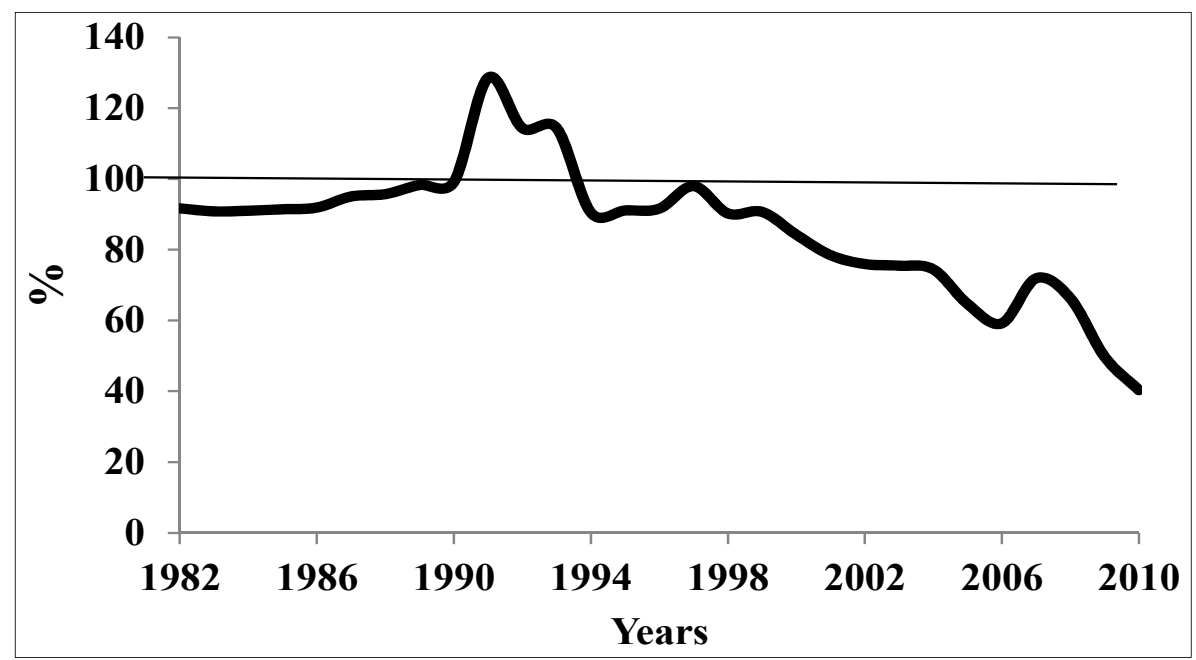

Source: Arab Organization for Agricultural Development, Statistical Yearbook (1982-2012) [3].

Figure 2. Total fish catch originating from Abu-Dhabi Emirate, UAE (2001-2010).

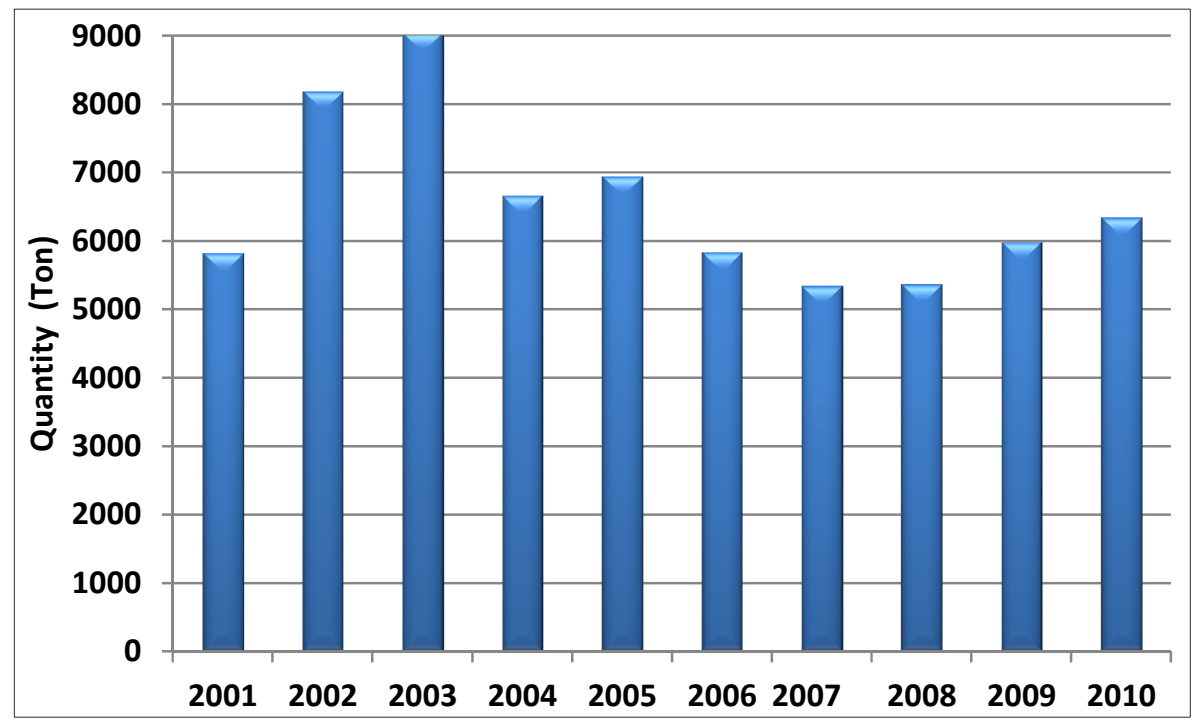

Source: Abu-Dhabi Environment Agency [5]. 
Since 2004, the total fish catch has stayed below the level of 7000 metric tons annually in the Emirate of Abu-Dhabi after the annual increase up to 2003 (Figure 2). The changes in the UAE fishing industry over the past three decades have increased uncertainty regarding sustainability (i.e., quantity and quality) of fish caught and consumed. The primary UAE fishing objectives are to maintain adequate sources of protein options for UAE consumers and to satisfy increasing consumer demand for healthier seafood products. Increasing fishermen resource use efficiency through the use of less risky fishing management practices may help contribute towards attaining UAE food security objectives.

Efficiency differences in fisheries are often attributed to differences in technology and the skill of the skipper and crew, as shown in Kirkley et al. [6] and Sharma and Leung [7]. However, Pascoe and Coglan [8] concluded that skipper skill is the major factor affecting differences in efficiency, with other factors explaining relatively little of the efficiency differences. Sherif and Gheblawi [9] examined the revenue determinants and assessed the input allocative efficiency of Abu-Dhabi fishermen. The main determinants of fishermen revenues were found to be labor, fishing effort, boat capacity, boat boarding expenses and expenditures on oil and lubricants. Moreover, price efficiency was found not to be achieved for any of the aforementioned five prominent inputs. More specifically, effort, boat capacity and labor were under-utilized, while boarding of the fishing crew and expenses on oil and lubricants were both found to be significantly over-utilized. Such inefficiency contributes towards increased risk and the decreased sustainability of the overall fishing industry in UAE. Sherif and Gheblawi [9] concluded that the increased risk faced by Abu-Dhabi fishermen causes declining revenue and leads fishermen to seek jobs in other sectors of the economy.

Aisyah et al. [10] used data envelopment and Tobit analysis to determine the technical efficiency level and factors influencing technical efficiency among Malaysian fishermen. The mean technical efficiency for the sample was estimated to be $55 \%$ for the peak season and $40 \%$ for the non-peak season. Variables related to management, such as planning, staffing and controlling, and variables related to demographics, such as family size and formal education, were found to exert positive effects on the technical efficiency of fisheries in Kuala Terengganu, Malaysia. Meanwhile, Ahmed et al. [11] used data envelopment analysis and also used Tobit models and found that integrated rice-fish farming was not popular among farmers in Bangladesh. The reasons for not adopting the technically efficient system were the lack of technical knowledge of farmers, high production costs and risks associated with floods and droughts. Espino et al. [12] utilized data envelopment analysis to study the capacity utilization of the fishing fleet in the Strait of Gibraltar. The results highlight the role increased technical efficiency has played in the persistence of overcapacity well after the implementation of recovery plans. The above group of studies addressed the technical efficiency of various fishery industries and analyzed fishermen socio-economic characteristics, which impact their technical efficiencies.

Singh et al. [13] developed a dynamic fishery model, which simultaneously incorporates random stock growth and costly capital adjustment, such that marginal net harvest benefits diminish with the catch (which provides an incentive to smooth harvest over time). The results demonstrate the importance of jointly determining harvest and capital investment. However, the value of the immediate harvest value rises as the fleet size increases. The results of Herrero and Pascoe [14] support the idea that the objective of fishermen is to maximize the value of the catch per trip rather than the quantity, while fishing strategies are affected by the presence of risk and uncertainty. Targeting high-value species by technically-efficient fishermen was found to be a more beneficial tactic than aiming for a 
high quantity catch of low-value fish species. In contrast, when the uncertainty of catching high-value fish is high, this tactic becomes costly to the inefficient fisherman. Danielsson [15] utilized a general bio-economic model to analyze the efficiency of different methods of fisheries management when there are some risks involved. The two management practices were the volume of catch in a catch-quota system and the volume of effort in an effort-quota system. It was found that when the price elasticity of demand is high and the variability in the growth of the stock is great compared to the variability in the catch per unit of effort (CPUE), management with effort quota was superior to management with catch quotas. However, if the price-elasticity of demand is low and the variability in the CPUE is large compared to the variability in the growth of the stock, management with catch quotas is superior to management with effort quotas.

Singh et al. [13], Herrero and Pascoe [14] and Danielsson [15] considered capital costs, the value of the catch and various fishing management alternatives, respectively. This group of studies considered the fishermen profit maximization problem under risk and uncertainty and discussed various fishery management alternatives. Bastardie et al. [16] developed a spatially explicit individual-based model for fishing vessel movements. The factors used in the model included vessel-based fuel consumption, energy efficiency defined as the quantity of fish caught per liter of fuel used and profitability. Fishermen adaptation to these factors was evaluated against three alternative effort allocation scenarios: (a) preferring nearby fishing grounds rather than distant grounds with potentially larger catches and higher values; (b) shifting to other fisheries targeting resources located closer to the harbor; and (c) allocating effort towards optimizing the expected area-specific profit per trip. The trade-off between fuel savings and energy efficiency improvements when effort is displaced closer to the harbor compared to reductions in total landing amounts and profit were the outcomes of Scenarios $\mathrm{a}$ and $\mathrm{b}$. Scenario $\mathrm{c}$ indicated that the historical effort allocation was actually sub-optimal, because increased profit from decreased fuel consumption and larger landings could have been obtained by applying a different spatial effort allocation. Data envelopment analysis was utilized by Maravelias and Tsitsika [17] to estimate and assess capacity utilization and efficiency in the Eastern Mediterranean. Longer vessels were found more efficient than shorter ones, and there was evidence of overcapacity. It was found that fishing vessels could potentially improve earnings by approximately $20 \%$ by increasing variable inputs. Andersen [18] used data on Danish seiners and data envelopment analysis to estimate efficiency and by accounting for fish stock development. The findings showed that estimations based on the catch per unit effort stock measures differ from the estimations based on independent stock measures and are independent of the choice of time horizon and the choice of input/output measures. Kirkley et al. [6] provided a comparative analysis of the deterministic data envelopment analysis and the stochastic production frontier capacity estimates for a variety of possible capacity concepts using a panel data set. The capacity output measures from data envelopment analysis were higher than the corresponding stochastic production frontier measures. However, the two approaches provided similar guidance about overall and even relative boat-specific capacity levels under certain circumstances.

Tingley et al. [19] compared the results from both data envelopment analysis and stochastic production frontier techniques for segments of English Channel fisheries. The factors most affecting technical efficiency were found to include vessel and skipper characteristics, and the results from both methods showed general consistency. The age of the vessel in the English Channel was found by 
Pascoe and Coglan [8] to be the most important measurable factor affecting efficiency. Immeasurable factors accounted for about $65 \%$ of the variation in efficiency and as much as $9 \%$ of the total variation in catches between boats. The factors contributing the most to those differences were found to be skipper and crew skills; however, "luck" accounted for around $11 \%$ of the variation in catches between boats. Del Hoyo et al. [20] estimated four econometric models measuring the technical efficiency of vessels operating in the Gulf of Cadiz, Spain. Since the approaches provided different estimates, the authors argued for different policy implications depending on the estimates adopted. They concluded that the useful life of vessels was inversely proportional to the technical efficiency, and the total allowable catch regime based only on vessels larger than $12 \mathrm{~m}$ was unsuitable for that particular fishery. Greenville et al. [21] utilized a stochastic frontier to examine the technical efficiency in input-controlled fisheries exemplified by the New South Wales (NSW) ocean prawn trawl fishery. They showed that input controls had a negative effect on the technical efficiency of fishermen, while such controls did not appear to have had a lasting effect on productivity.

The above studies show the importance of decision-making in relationship to different management alternatives at the fishermen decision-maker (DM) level under various risk and uncertainty conditions. Successful fishing practices were found to be those that considered the impact of boat capacity, fishing effort, size of capital investment, boat length, labor training and management, as well as the number and sizes of traps. Previous studies also highlighted the importance of diversification, economies of scale and the catch of high-value fish management options on fishing profitability and both technical and economic efficiency.

The overall goal of this research is to address concerns related to risk and uncertainty in the fishing industry in the Emirate of Abu-Dhabi, UAE. The specific objective of this paper is to quantify and rank UAE fishermen preferences concerning various fishing management alternatives at different levels of attitudes towards risk. To achieve the specific research objective, several statistical and stochastic analysis techniques were employed, including descriptive statistic and stochastic dominance techniques (first/second degree stochastic dominance, stochastic dominance with respect to a function and stochastic efficiency with respect to a function) to rank the fishing management alternatives (methods of fishing, sizes of traps and number of traps) based on a well-accepted economic indicator (profitability represented by gross margin). The stochastic dominance techniques were applied across a spectrum of risk attitudes and preferences ranging from risk neutral to risk averse. The significance of this research stems from the DM need to identify less risky management alternatives, the adoption of which will hopefully retain more UAE fishermen in the business, attract a new generation of fishermen and augment the sustainability of this important food sector to ensure a sufficient and long-term supply of fish in the UAE.

This paper is divided into four sections. The first section summarized the overall UAE fisheries' socio-economic indicators, a literature review of previous studies and the research objective, which focuses on quantifying and ranking UAE fishermen preferences concerning various fishing management alternatives at different levels of attitudes towards risk. The second section of this paper describes the methods used, including stochastic first and second degree stochastic dominances, stochastic dominance with respect to a function and stochastic efficiency with respect to a function, to assess the risk facing UAE fishermen. The third section includes the results and discussion, starting with the analysis of the Abu-Dhabi fishermen survey results and continues with the ranking of the 
fishermen management alternatives using various ranking methods. The fourth section of this paper includes a summary and conclusion, which summarizes the importance of considering both decision-maker's attitude towards risk and economic inefficiencies in managing UAE fisheries that can be used for designing successful fisheries policies.

\section{Methods}

\subsection{Standard Stochastic Dominance Techniques}

In economic systems, there is generally risk associated with production quantities, input prices, output prices, interest rates, quantities demanded by consumers, market share, government regulations and weather conditions, among others. For a business, a manager does not have control over many of these variables, i.e., risk is the part of a business decision the manager cannot control. Risk is often quantified using probability theory, i.e., a stochastic process involving random variables that is often used to represent the evolution of a system over time or space. Stochastic dominance is a methodology based on expected utility maximization theory. A detailed discussion of the usefulness of stochastic dominance decision criteria can be found in Robison and Barry [22]. Boggess and Ritchie [23] and Williams et al. [24] also present the rationale and application of various techniques. The theoretical attractiveness of stochastic dominance analysis lies in its non-parametric orientation, i.e., it does not require a full parametric specification of the preference of the decision-maker and the statistical distribution of the choice alternative [25-27].

As previously discussed, several well-known decision criteria for stochastic dominance exist, including first degree stochastic dominance (FSD), second degree stochastic dominance (SSD) and stochastic dominance with respect to a function (SDRF). Hardaker et al. [28] stated that FSD assumes that the decision-maker (DM) has positive marginal utility for the performance measure (i.e., more is preferred to less). Thus, given two alternatives, A and B, each with a probability distribution of outcomes defined by a Cumulative Distribution Function (CDF), A dominates B in the FSD sense if the $\mathrm{CDF}$ of $\mathrm{A}$ is always below and to the right of the $\mathrm{CDF}$ of $\mathrm{B}$ (i.e., $\mathrm{FA}(\mathrm{X}) \leq \mathrm{FB}(\mathrm{X})$ for all $\mathrm{X}$ ), where $\mathrm{F}(\mathrm{X})$ denotes the distribution describing the random variable $(\mathrm{X})$. SSD holds for those decision makers who are risk neutral or risk averse for all values of $\mathrm{X}$; thus, the applicable range of the absolute risk aversion coefficient, $r_{a}$ (a measure of how much a person would pay to avoid risk), for the SSD criterion is from 0 to $+\infty$. SSD assumes a utility function of a positive, but decreasing slope, i.e., $\mathrm{U}^{1}(\mathrm{X})$ $>0$ and $\mathrm{U}^{2}(\mathrm{X})<0$ with $\mathrm{SSD}$, and $\mathrm{A}$ is preferred to $\mathrm{B}$ if $\int_{-\infty}^{x} F_{A}(X) d x \leq \int_{-\infty}^{x} F_{B}(X) d x$ for all values of $X$. Graphically, this means SSD requires that the curve of the cumulative area under the CDF for a dominant alternative lies everywhere below and to the right of the corresponding curve for the dominated alternative. Although more powerful than FSD, SSD often leaves a large number of choices as being risk-efficient. To improve the discriminating power of SSD, Meyer [29] proposed SDRF, which is a more general notion of stochastic dominance. This rule helps to identify risk-efficient options for the class of decision-makers whose risk aversion coefficients are bounded by lower and upper values. The smaller the range of risk aversion coefficients, the more powerful is the criterion. The SDRF criterion orders the choices by defining intervals using the $r_{a}$ absolute risk aversion coefficients. These risk-preference intervals are bounded by a lower risk aversion coefficient, $\mathrm{r}_{\mathrm{aL}}$, and 
an upper risk aversion coefficient, $\mathrm{r}_{\mathrm{aU}}$, which characterize the general degree of risk aversion for a manger. A risk-efficient set of strategies will include the choices preferred by each manager having risk preferences consistent with the restrictions imposed by the lower to upper interval. Furthermore, Hardaker et al. [30] argued that eliciting decision-makers (or inferring) the bounds on their risk aversion coefficients may be simpler than eliciting a complete utility function. Further discussion of SDRF is provided by Cochran [31], King and Robison [32] and Robison and Barry [24].

\subsection{Stochastic Efficiency with Respect to a Function (SERF)}

A more discriminatory procedure than SDRF that considers the full range of decision-maker preferences is stochastic efficiency with respect to a function (SERF). SERF is more efficient than other stochastic dominance techniques, because it can be applied for any utility function form based on the full range (i.e., from negative to positive) of an absolute or relative risk aversion coefficient $\left(r_{a}\right)$. A positive $r_{a}$ indicates that the DM is risk averse, while a negative $r_{a}$ indicates that the DM is a risk taker (a risk-neutral DM is more concerned about the expected return on their investment, not on the risks they may be assuming). Furthermore, SERF is a preferable method, due to the fact that it is one of few risk analysis techniques that can be used to easily visualize the stochastic frontier across the entire $r$ range, where preferences for a particular alternative may be illustrated.

SERF identifies and orders utility efficient alternatives in terms of certainty equivalents (CEs) for a specified risk preference. Hardaker et al. [28] state that the SERF procedure can potentially find a smaller set of preferred strategies (i.e., it has stronger discriminating power) compared to stochastic dominance approaches in addition to being more transparent and easier to implement. The $\mathrm{CE}$ of a risky alternative (in this study, the trap, net and thread fishing methods) is the amount of money at which the decision-maker is indifferent between the certain dollar value and the risky alternative. That is, the $\mathrm{CE}$ is the sure amount of money with the same utility as the expected utility of a risky alternative as suggested by Keeney and Raiffa [33] and can be calculated by taking the inverse of the utility function, $\mathrm{U}$ :

$$
\mathrm{CE}(\mathrm{w}, \mathrm{r}(\mathrm{w}))=\mathrm{U}^{-1}(\mathrm{w}, \mathrm{r}(\mathrm{w}))
$$

where $w$ is the initial wealth and $r(w)$ represents the risk aversion coefficient with respect to wealth. The utility function, $U$, can be represented as:

$$
\mathrm{U}(\mathrm{w}, \mathrm{r}(\mathrm{w}))=\int \mathrm{u}(\mathrm{w}, \mathrm{r}(\mathrm{w})) \mathrm{dF}(\mathrm{w})
$$

where $U(w, r(w))$ is the utility as a function of the random variable, $w$, and a set of risk aversion coefficients, r. Strategies with higher CEs are preferred to those with lower CEs, and the interpretation of the CEs is straightforward, because, unlike utility values, they may be expressed in monetary terms [34]. To calculate the CEs using SERF, various types of utility functions can be used (e.g., power, negative exponential, quadratic, log-log). In this study, similar to that of Pendell et al. [34], we assume a negative exponential form for the utility function:

$$
U(w)=-\exp \left(-r_{a}(w) w\right)
$$


where $r_{a}(w)$ is the absolute $r_{a}$ with respect to wealth. Given a random sample of size $\mathrm{n}$ from alternative $\mathrm{w}$ (i.e., a management alternative, such as fishing method) with i possible outcomes (i.e., the fisheries' gross margin), the estimated CE can be defined as:

$$
C E\left(w, r_{a}(w)\right)=-\frac{1}{r_{a}(w)} \ln \left\{\frac{1}{n} \sum_{i}^{n} \exp \left(-r_{a}(w) w_{i}\right)\right\}
$$

A negative exponential utility function conforms to the hypothesis that managers prefer less risk to more given the same expected return and assumes managers have constant absolute risk aversion, as suggested by Lien et al. [34]. Under this assumption, managers view a risky strategy for a specific level of risk aversion the same without regard for their level of wealth, such as in Pendell et al. [35], and Babcock et al. [36] state that this functional form is often used to analyze decisions-maker's alternatives under risk. The decision rule for SERF is to rank the risky alternatives (within the decision-makers' specified risk aversion coefficient) from the most preferred (i.e., the highest CEs at specified levels of risk aversion) to the least preferred (i.e., the lowest CEs at specified levels of risk aversion). There is a general agreement on the valid range for the $r_{a}$ that a DM should use with SERF [37].

In this study, UAE fishing management alternatives (methods, size of traps and number of traps) were considered for the descriptive statistic and stochastic dominance analyses. Fishermen catch determines revenues, because the fishermen are a price taker in the market. Subtracting the cost of operations from revenues determines the gross margin or revenue over variable cost. The fishing management alternatives are analyzed under various levels of fishermen attitudes towards risk. SERF is a practical method for analyzing the fishing management alternative problem, because SERF considers both the variability of gross margins and DM attitude towards risk. As stated above, SERF calculates a CE, which is the sure amount of money with the same utility as the expected utility of a risky alternative [37].

SERF is a procedure for ranking risky alternatives based on the certainty equivalent (CE) for risk alternatives and the absolute risk aversion coefficients (ARAC). Hardaker et al. [28] described three steps that are considered the computation steps for the SERF discrete CE points. In the first step, selected points on each CDF from the set of $w$ values are determined. Then, the second step is to convert the selected $w$ values to their utility function (i.e., the negative exponential function in this study) value, as well as the values representing the risk aversion coefficient are selected. The third step involves multiplying each finite utility by its corresponding probability to calculate the weighted average of the utility outcome. As a result, only alternatives that have higher CE values for some specified range of $\mathrm{r}(w)$ are utility efficient. The Simetar@ 2008 risk analysis software implements the steps above, and they are graphically illustrated in the software's user manual [37]. The next section in this paper further describes the stochastic dominance and stochastic efficiency analyses adopted for this paper.

\subsection{Risk Simulation Analyses}

The Simetar (C) 2008 risk analysis software [37] was used to perform the stochastic dominance (FSD, SSD and SDRF) and SERF analyses. For the SDRF stochastic dominance analysis, two points represented the spectrum of decision-maker attitude towards risk. A lower absolute risk aversion coefficient $\left(\mathrm{r}_{\mathrm{aL}}\right)$ equal to 0.0 (representing a neutral decision-maker) and an upper absolute risk 
aversion coefficient $\left(\mathrm{r}_{\mathrm{aU}}\right)$ equal to 0.004 (representing a risk-averse decision-maker) were used to perform the analysis. For the SERF analysis, gross margin CE curves by fishing management alternatives (fishing methods, size of traps and number of traps) were produced by calculating $25 \mathrm{CE}$ values for each curve over the entire range (0.0 to 0.004) of absolute risk aversion $\left(r_{a}\right)$. Stochastic efficiency with respect to a function (SERF) is considered a sufficient ranking method. Hardaker [30] showed that SERF will potentially identify a smaller efficient set than SDRF, because it picks only the utility efficient alternatives, compared to other alternatives simultaneously, at each point of the DM attitude towards risk.

\subsection{Study Data Source}

The data for this study were extracted from face-to-face interviews with Emirate of Abu-Dhabi, UAE, fishermen. The United Arab Emirates Ministry of Water and Environment (MOEW) [38] showed that the Emirate of Abu-Dhabi area was targeted because of the on-going intensive fishing activities taking place across the Emirate's shores. The number of boats increased from 856 in 2001 to 1100 in 2009 or about 30\% [38]. One hundred thirty one fishermen (representing about 9\% of the total population of fishermen in the area) participated in the survey, which was divided into four sections. The first section focused on measuring the socioeconomic characteristics of the fisheries. The second section investigated the asset values (i.e., fixed costs) of the fisheries, such as boat market values, as well as equipment values. The third section collected data on variable costs, including the quantities of inputs used and input prices (e.g., labor, material and supplies costs). Finally, the fourth section investigated the output (fish catch) and revenue (fish catch multiplied by output prices) of the fisheries. Data from the survey were entered into a spreadsheet, coded and prepared for analysis. For complete details of the survey, see Sherif and Gheblawi [9].

\section{Results and Discussion}

\subsection{Fisheries Survey Descriptive Statistics}

Based on the 131 fishermen interviewed, the survey sample results indicated that the average fishing boat age was about 22 years with a standard deviation (SD) of nine years. The average fishing boat market price was 142,000 Dirhams (AED) with an SD of 93,000 Dirhams. The United Arab Emirate Dirham (AED) is the official currency of the UAE (one USD = 3.675 AED). A boat license costs UAE fishermen about 220 AED on average, and survey results showed that there was on average four workers per boat earning a salary of 712 AED per worker monthly. The average boat capacity was around 208 horsepower with an SD of 67.5 horsepower; the average boat length was 32 feet $(9.8 \mathrm{~m})$ with an SD deviation of 14.4 feet $(4.4 \mathrm{~m})$. The number of fishing trips per month ranged from two to 22 trips. On average, each fisherman went on six fishing trips per month, with each trip taking four days on average. Investigation of the socio-economic characteristics of Abu-Dhabi fishermen showed that $97 \%$ of the fishermen were boat owners with only $3 \%$ renting boats for fishing. The survey results showed that $53 \%$ of the fishermen were UAE nationals, while $47 \%$ were citizens of other countries (e.g., India, Iran and Oman). When survey respondents were asked if they purchased boat insurance, only nine fishermen (or 7\%) responded yes. Furthermore, only 37 survey respondents (or 28\%) 
indicated that their sons were interested in pursuing fishing as a profession and career. These findings confirm the fact that the next generation does not consider fishing in Abu-Dhabi as a secure source of income (and would not want to enter the business and sustain it for the future). Five different species of fish were identified as common in the Abu-Dhabi fishermen daily catch, including Hamour, Kan'ad-Chabbat, Sultan Ibrahim, and Safi Arabi. However, Abu-Dhabi fishermen are not specialized in fishing of a certain species in favor of other species. Their daily catch is mostly a mix of different species based on stock availability and seasonal fish migration. Only 18 survey respondents (or 14\%) were found to target and find shrimp in their catch.

The survey results also focused on the estimation of the gross margin per boat on an annual basis. The gross margin was calculated by subtracting the variable costs, which are costs that vary with the quantity of the fish catch and include the costs of labor, fuel, maintenance and boarding (such as food, nets, water and ice). Labor and fuel costs combined represent $89 \%$ of the total variable costs for an average Abu-Dhabi fisherman. Labor and fuel represent $62 \%$ and $27 \%$ of the variable costs, respectively. Laborers are commonly foreigners; it costs boat owners about 4370 AED on average to hire a worker from abroad. The economic results of the Emirate of Abu-Dhabi, UAE, fishermen survey are presented in Table 1. The average annual total revenue is about 298,000 AED, subtracting average annual variable costs of 159,000 AED from the total revenue results in an average gross margin of 139,000 AED. The SD of the total revenue, annual variable costs and gross margin is $186,000,52,000$ and 174,000 AED, respectively. The higher SD for the gross margin reflects the large economic variability in the survey sample. The coefficient of variation $(\mathrm{CV}$, calculated as the SD divided by the mean) is a more accurate measure of variability compared to the SD. The annual gross margin $\mathrm{CV}$ of the sample is 1.25, which indicates that the SD is higher than the mean for the Abu-Dhabi, UAE, fisheries. The total revenue, variable costs and gross margin standard deviations in Table 1 are high, which indicate the large variability of both input costs and revenue. This finding indicates the need to use an alternative quantitative approach (e.g., stochastic dominance analysis) to further investigate the variability and the overall impact of the various fishing management practices on the gross margin.

Table 1. Descriptive statistics of selected economic variables based on the Emirate of Abu-Dhabi, UAE, fisheries survey.

\begin{tabular}{lccc}
\hline \multicolumn{1}{c}{ Variable } & $\begin{array}{c}\text { Annual total } \\
\text { revenue (TR) } \\
\text { (AED) }\end{array}$ & $\begin{array}{c}\text { Annual variable } \\
\text { costs (VC) } \\
\text { (AED) }\end{array}$ & $\begin{array}{c}\text { Annual gross margin (GM) } \\
\text { = TR - VC (AED) }\end{array}$ \\
\hline Mean & 298,173 & 159,112 & 139,061 \\
Median & 276,000 & 157,680 & 125,320 \\
Standard deviation (SD) & 185,899 & 52,415 & 174,042 \\
Coefficient of variation (SD/mean) & 0.62 & 0.33 & 1.25 \\
Number of observations & 131 & 131 & 131 \\
\hline
\end{tabular}

Table 2 summarizes descriptive statistics for the gross margin (total revenue (TR) - variable costs (VC)) for the different fishing methods in the Emirate of Abu-Dhabi, UAE. Based on the mean, the traps method had the highest average gross margin (about 168,000 AED annually) compared to the thread and net methods. However, the higher average for the traps method was also accompanied by the highest standard deviation (about 176,000 AED) for all of the fishing alternatives. The CV results, 
commonly used to indicate risk, show that the most risky alternative would be the thread method of fishing. In theory, a manager could examine only the mean standard deviation and CV risk-tradeoff results and simply decide which fishing method alternative is best; however, this may be difficult in practice in that these criteria often exhibit high variability and can result in contradictive and inconclusive rankings. The application of a more sophisticated risk-based methodology, such as stochastic dominance or SERF, can help fishermen and decision-makers see these tradeoffs more clearly with very few additional assumptions.

Table 2. Descriptive statistics of annual gross margins (AED) for various methods of fishing based on the Emirate of Abu-Dhabi, UAE, fisheries survey.

\begin{tabular}{lccc}
\hline Statistical measure & Traps & Thread & Nets \\
\hline Mean & 167,551 & 65,078 & 67,274 \\
Median & 159,360 & 66,430 & 45,280 \\
Standard deviation (SD) & 175,891 & 169,834 & 142,885 \\
Coefficient of variation (SD/mean) & 1.05 & 2.61 & 2.12 \\
Number of observations & 94 & 10 & 27 \\
\hline
\end{tabular}

\subsection{First Degree Stochastic Dominance (FSD)}

First degree stochastic dominance (FSD) can be determined by visually considering the Cumulative Distribution Functions (CDFs) between various management alternatives. As previously stated, Alternative A completely dominates Alternative B if the CDF of Alternative A is always below and to the right of the CDF of $\mathrm{B}$ (i.e., the CDFs do not intersect). Figures 3-5 depict Abu-Dhabi, UAE, fisheries' annual gross margin CDFs for the fishing method, trap size and number of traps, respectively, based on the fisheries sample. All three graphs show inconclusive results that do not show a dominant alternative, because all CDFs cross each other either at the upper or the lower tails of the CDF. In Figure 3, the CDF for the trap fishing method is further to the right compared to the CDFs for the thread and net fishing methods. However, the trap method CDF crosses the net method CDF in the upper tail (when the decision-maker is more risk averse). Hardaker [30] showed that if CDF A crosses $\mathrm{CDF} \mathrm{B}$, where $\mathrm{A}$ and $\mathrm{B}$ are the probability distributions of the outcome, then neither dominates the other in the first degree sense. In other words, $\mathrm{A}$ dominates $\mathrm{B}$ only if $\mathrm{F}_{\mathrm{A}}(x) \leq \mathrm{F}_{\mathrm{B}}(x)$ with at least one strong inequality. Figures $3-5$ below indicate that no clear dominance of the fishing management alternatives exists. The absence of a dominant alternative for the fishing method, trap size or number of traps necessitates conducting a second degree stochastic dominance analysis.

Figure 4 shows FSD results for the gross margin based on trap size, and Figure 5 shows FSD results for the gross margin based on the number of traps used by fishermen in Abu-Dhabi. Again, both figures show non-conclusive results, because the CDFs intersect at the upper tails of the graph. These results indicate that the DM cannot rely on FSD to rank the risky alternatives.

To further investigate the statistical differences between the three sets of management alternative distributions (fishing methods, traps sizes and number of traps), Kolmogorov-Smirnov tests were performed with results reported in Table 3. The test results showed no significance differences between cumulative probability distributions for fishing method (traps, threads and sets) pairs. Similar to fishing methods, no significance differences were found between cumulative probability 
distributions for the number of trap pairs. However, the Kolmogorov-Smirnov test showed the presence of significant differences between the cumulative probability distributions for trap size (large, medium and small). These results are different than those obtained from the visual inspection of the CDFs (FSD) in ranking the risky management alternatives. The analysis proceeds using second degree stochastic analysis in the search for more conclusive stochastic analysis results.

Figure 3. Sample annual gross margin (AED) cumulative distribution functions (CDFs) for different methods of fishing.

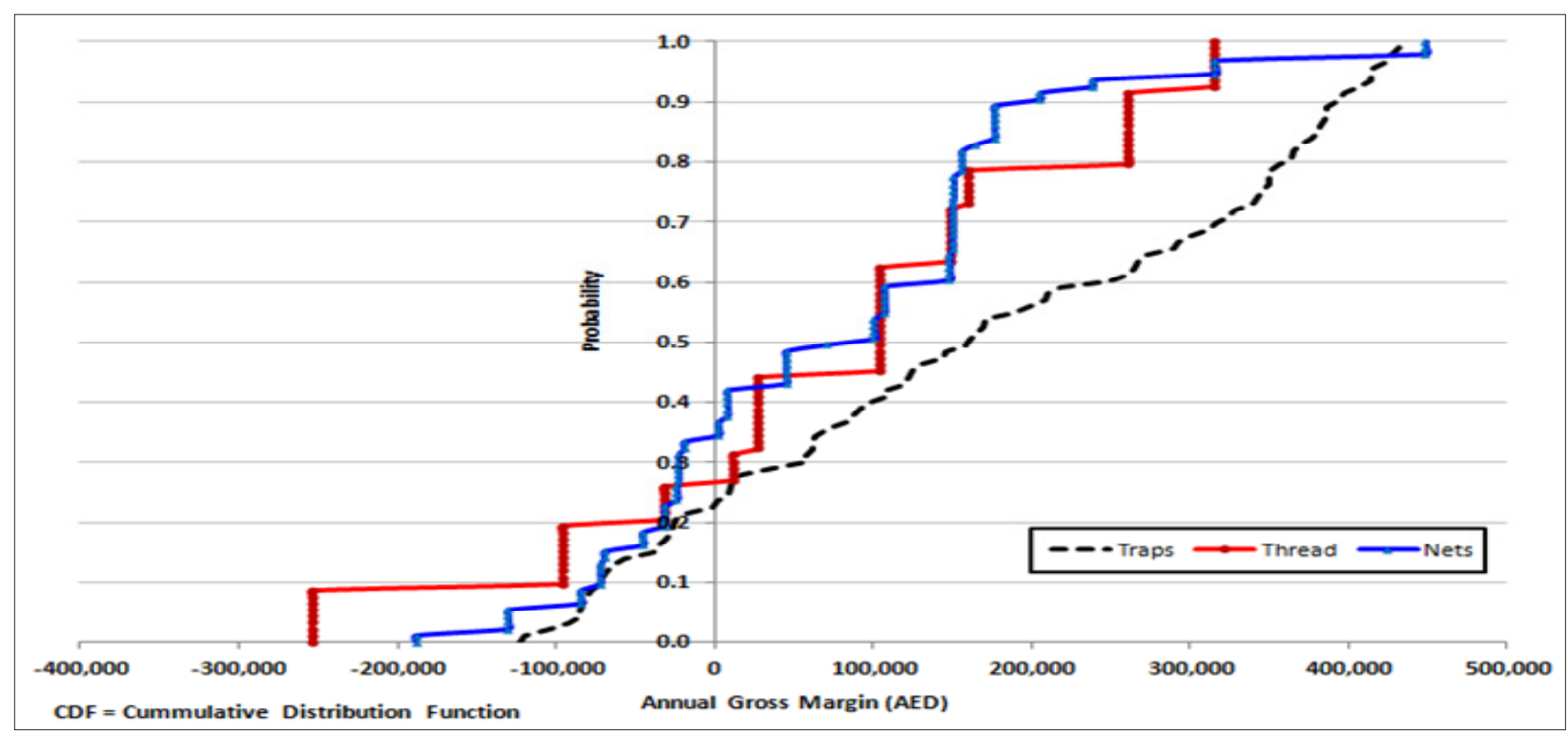

Figure 4. Sample annual gross margin (AED) cumulative distribution functions (CDFs) for trap sizes.

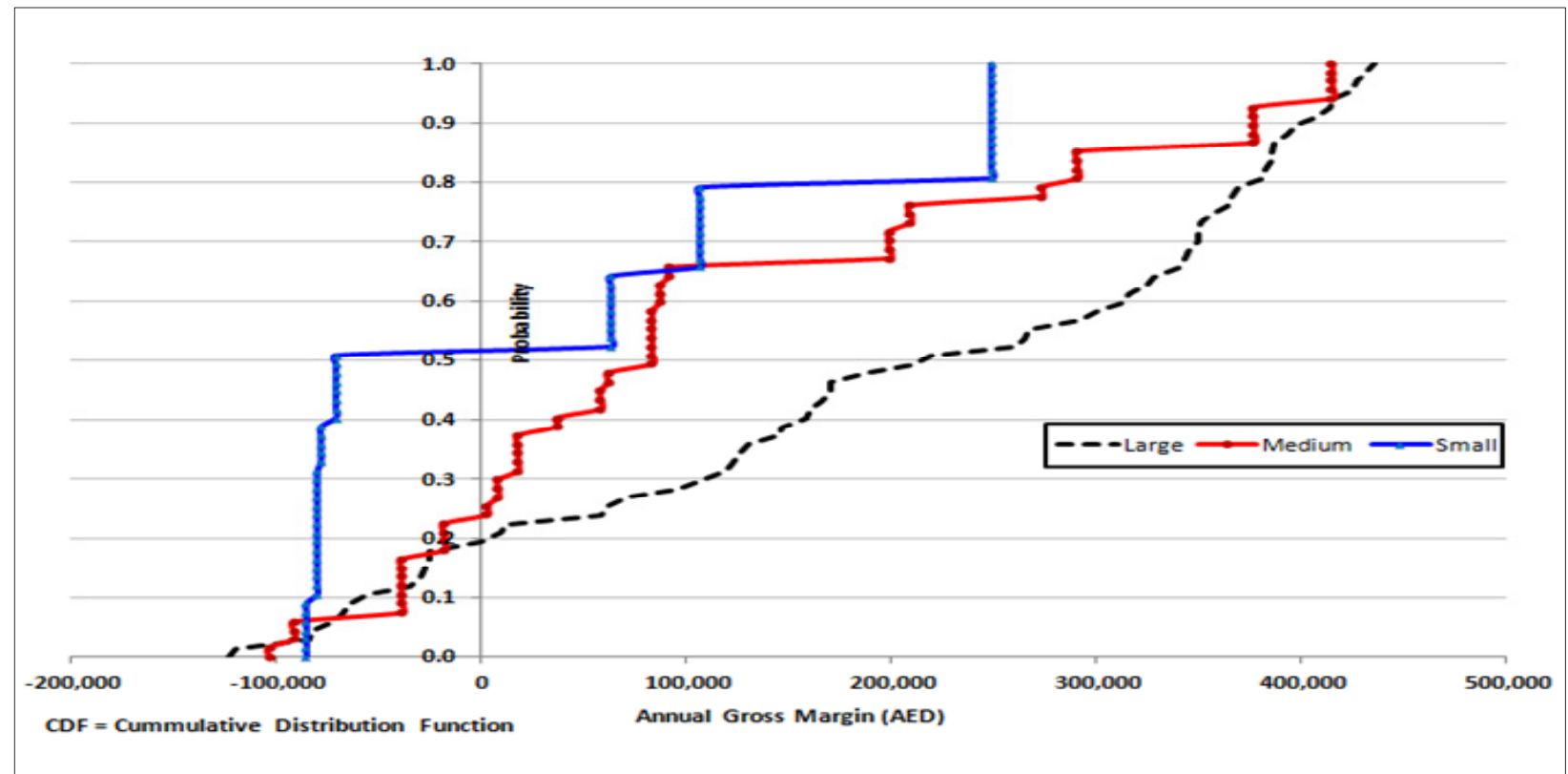


Figure 5. Sample annual gross margin (AED) cumulative distribution functions (CDFs) for the number of traps.

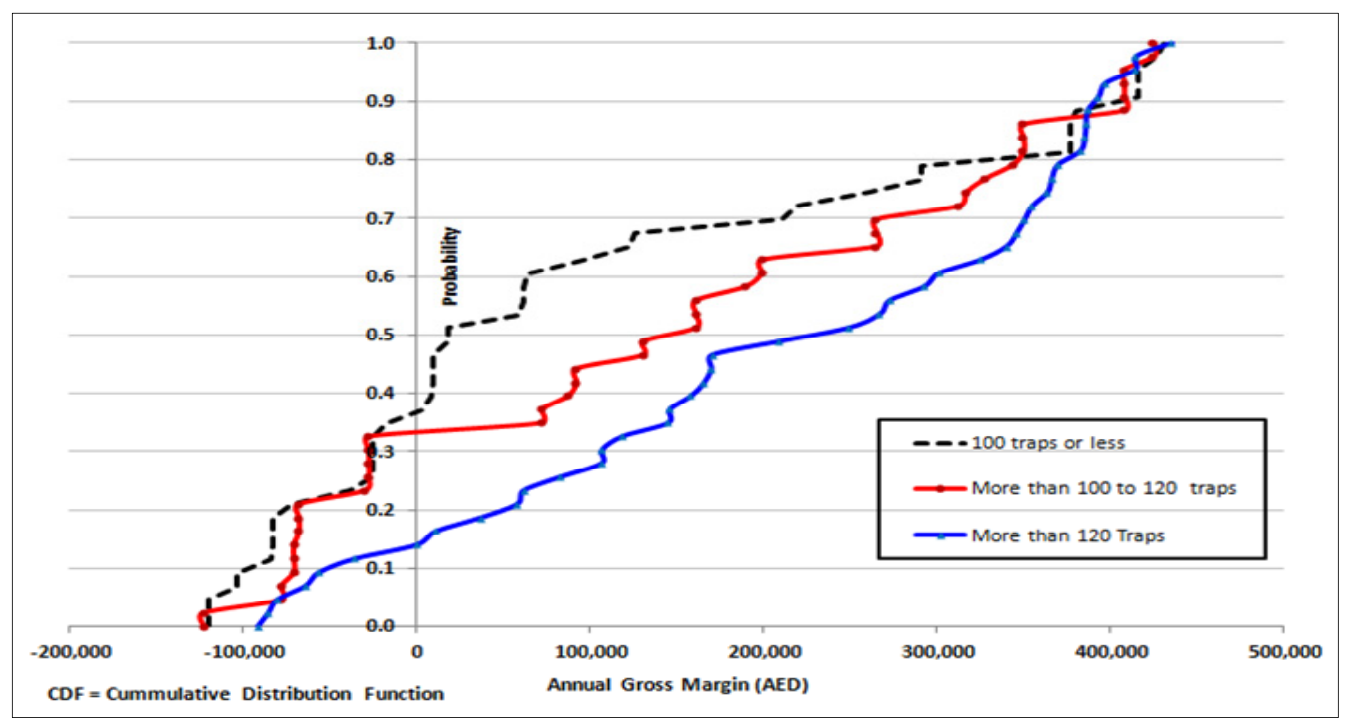

Table 3. Kolmogorov-Smirnov (K-S) statistical significance tests of cumulative distribution functions (CDFs) for pairs of fishing management alternatives (methods, size of traps and number of traps).

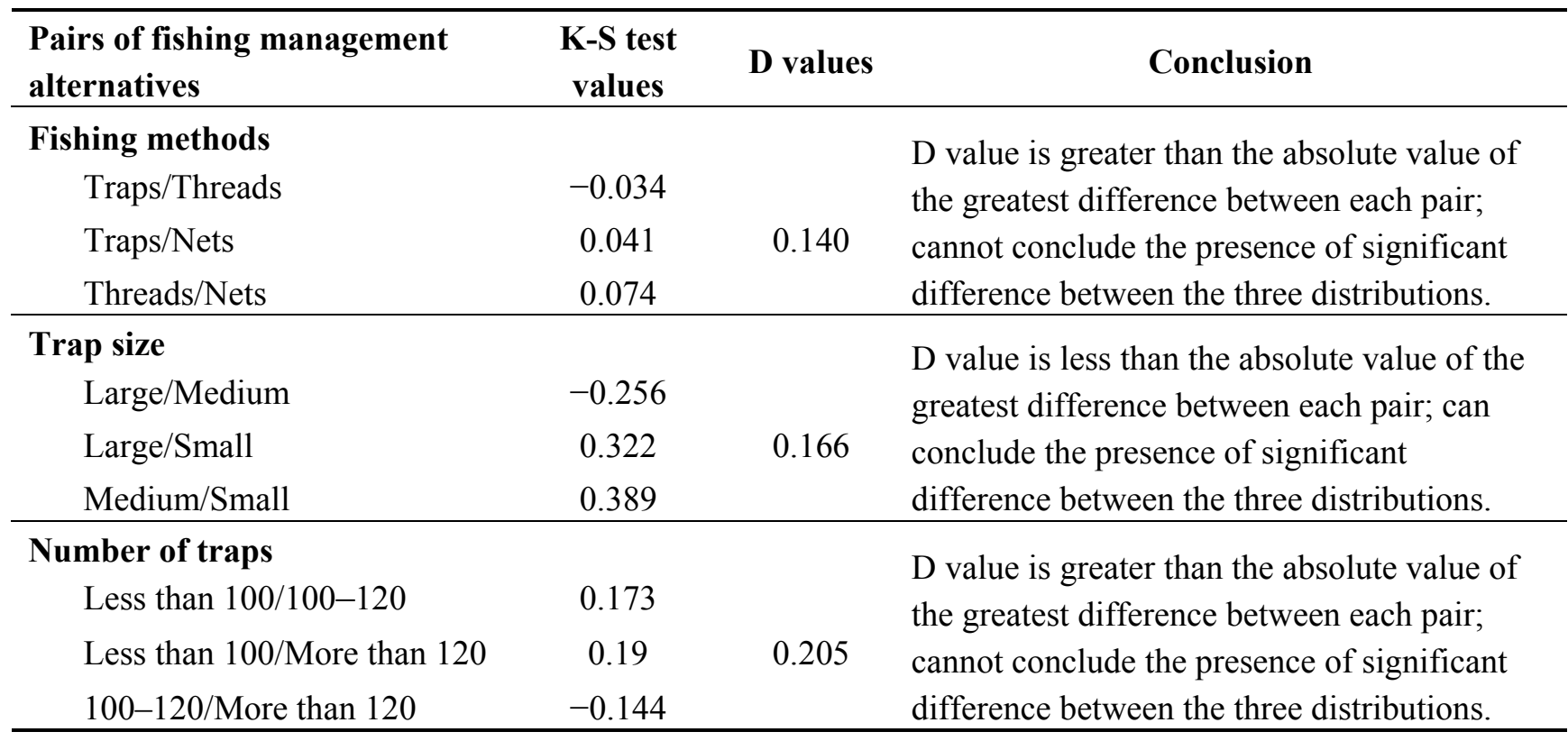

\subsection{Second Degree Stochastic Dominance (SSD)}

Table 4 below illustrates the second degree stochastic dominance (SSD) results for methods of fishing, types of traps and numbers of traps, respectively, based on the gross margin. SSD results indicate that fishery operation profitability risk is higher when fishermen are using threads and nets. The risk is also higher when fishing operations use medium- and small-sized traps and less than 120 traps. The SSD results indicate that: (1) the trap fishing method dominates both the thread and the net methods; (2) large-sized traps are dominant over medium- and small-sized traps; and (3) using more than 120 traps dominates the lesser number of trap alternatives. 
Table 4. Second degree stochastic dominance (SSD): gross margins based on indicators for the methods of fishing, the size of traps and the number of traps.

\begin{tabular}{lllll}
\hline Fishing methods & Traps & Threads & Nets & SSD dominance ranking \\
\hline Traps & -- & Dominant & Dominant & 1 \\
Threads & Not Dominant & -- & Not Dominant & 3 \\
Nets & Not Dominant & Dominant & -- & 2 \\
\hline Trap size & Large & Medium & Small & SSD dominance ranking \\
Large & -- & Dominant & Dominant & 1 \\
Medium & Not Dominant & -- & Dominant & 2 \\
Small & Not Dominant & Not Dominant & -- & 3 \\
\hline \multirow{2}{*}{ Number of traps } & $\mathbf{1 0 0}$ traps & More than $\mathbf{1 0 0}$ & More than & SSD dominance ranking \\
& or less & to 120 traps & $\mathbf{1 2 0}$ traps & \\
100 traps or less & -- & Not Dominant & Not Dominant & 3 \\
More than 100 to 120 traps & Dominant & -- & Not Dominant & 2 \\
More than 120 Traps & Dominant & Dominant & -- & 1 \\
\hline
\end{tabular}

\subsection{Stochastic Dominance with Respect to a Function (SDRF)}

Stochastic dominance analyses include specific assumptions related to DM attitude towards risk. Table 5 summarizes the stochastic dominance with respect to a function (SDRF) results for fishing methods, trap size and number of traps. Among the various fishing methods, threads is preferred over other fishing methods (traps and nets) for both the risk-neutral and the extreme risk-averse DM. The risk-neutral DM, based on the SDRF risk analysis method, would select a large trap size over other sizes (medium, small). In contrast to the risk-neutral DM, the risk-averse DM would prefer small trap sizes over the large size, potentially to avoid large losses, as large trap sizes are usually accompanied by higher spending on inputs, such as labor and fuel, and require a larger catching effort. Considering the SDRF for risk analysis, the DM would prefer more than 120 traps over less than 120 traps for both risk-neutral and risk-averse conditions.

Table 5. Stochastic dominance with respect to a function (SDRF) rankings for the fishing management alternatives based on the Emirate of Abu-Dhabi, UAE, fisheries survey.

\begin{tabular}{|c|c|c|}
\hline Management alternatives & $\begin{array}{l}\text { Efficient set ranking with an absolute } \\
\text { risk aversion coefficient }=0.0\end{array}$ & $\begin{array}{l}\text { Efficient set ranking with an absolute } \\
\text { risk aversion coefficient }=\mathbf{0 . 0 0 4}\end{array}$ \\
\hline \multicolumn{3}{|l|}{ Fishing methods } \\
\hline Thread & Most Preferred & Most Preferred \\
\hline Traps & 2nd Most Preferred & 2nd Most Preferred \\
\hline Nets & 3rd Most Preferred & 3rd Most Preferred \\
\hline \multicolumn{3}{|l|}{ Trap size } \\
\hline Large & Most Preferred & 3rd Most Preferred \\
\hline Medium & 2nd Most Preferred & 2nd Most Preferred \\
\hline Small & 3rd Most Preferred & Most Preferred \\
\hline \multicolumn{3}{|l|}{ Number of traps } \\
\hline More than 120 traps & Most Preferred & Most Preferred \\
\hline More than 100 to 120 traps & 2nd Most Preferred & 2nd Most Preferred \\
\hline 100 traps or less & 3rd Most Preferred & 3rd Most Preferred \\
\hline
\end{tabular}




\subsection{Stochastic Efficiency with Respect to a Function (SERF)}

Figures 6-8 illustrate the stochastic efficiency with respect to function (SERF) results for the three ranking efficiency indicators (i.e., fishing methods, size of traps and number of traps). The advantage of SERF over the other risk analysis methods is that SERF enables a DM to rank the various alternatives at different levels of attitude towards risk. Figure 8 shows that a DM would prefer traps over threads and nets when the gross margin CE is used at all positive levels of attitude towards risk. In other words, across all ranges of attitudes towards risk, including risk neutral and risk averse, the DM would choose traps over the other fishing methods. Figure 6 also shows that the net alternative is dominant over the thread alternative. Figure 7 shows that the large trap alternative dominates the medium- and small-sized trap alternatives when the DM is risk neutral. This implies that the variability/stochastic impact is higher among fishermen who are using large traps compared to those who use medium- or small-sized traps. This stochastic impact is due to the fact that large traps are used by large boats, which are the ones that usually take longer and more frequent trips and, so, are exposed to relatively higher levels of uncertainty and variability.

Figure 6. Stochastic efficiency with respect to a function (SERF) for fishing methods (negative exponential utility function).

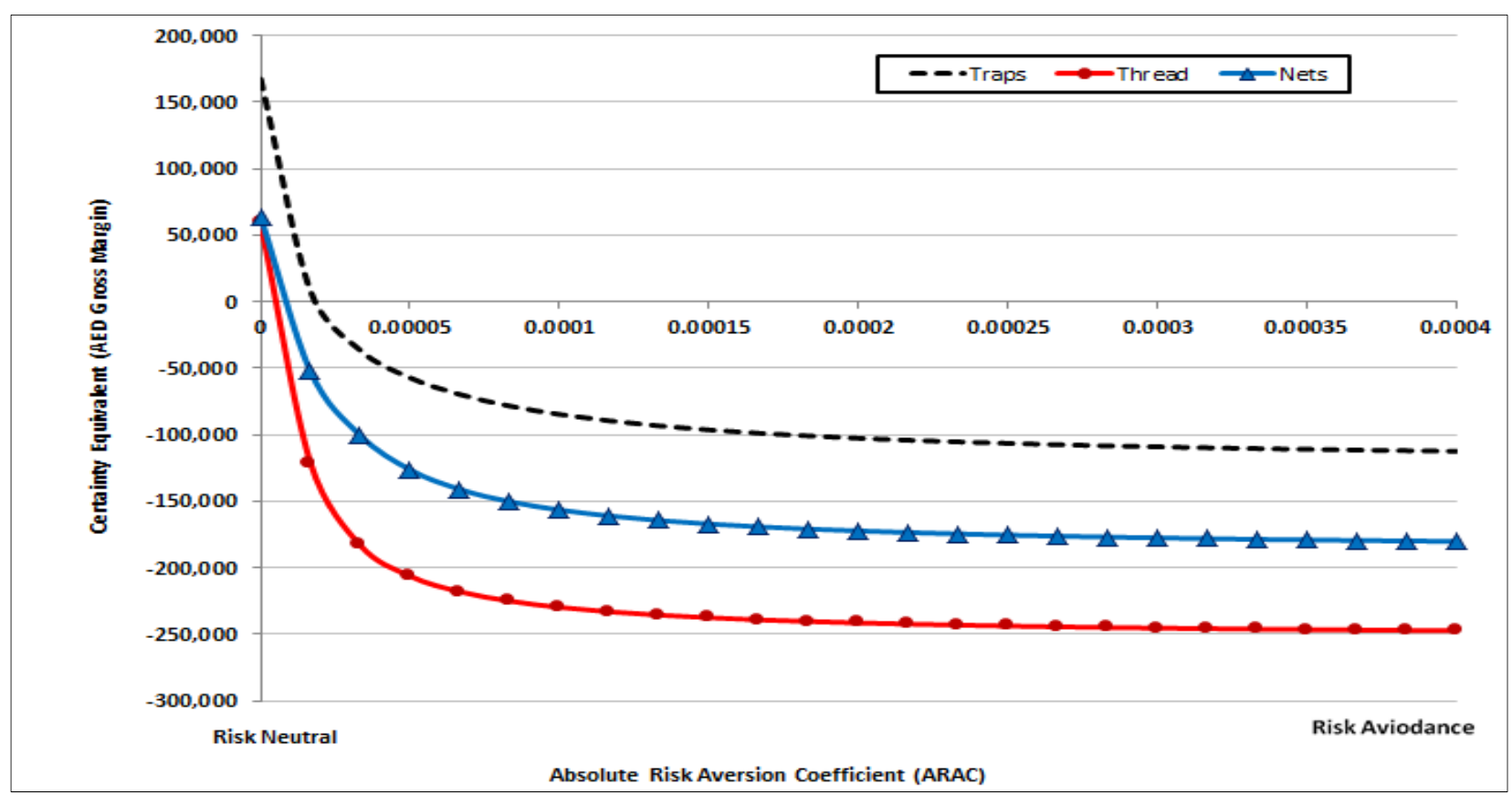

Figure 8 shows the SERF results based on the gross margin for the number of traps. Consistent with the SSD results, the SERF analysis indicates that using the more than 120 traps alternative is dominant over the smaller number of traps alternative across all levels of attitude towards risk. Using the stochastic efficiency approach and analysis reveals that using traps is more efficient than other fishing alternatives (threads and nets) and using large traps is more efficient than using medium or small traps. Furthermore, the analysis reveals that 120 traps or more is more efficient than using less than 120 traps. One could also conclude that the thread and net fishing methods are not efficient relative to using traps (i.e., they are more risky alternatives). Using medium and small traps are risky alternatives, as well, in addition to using less than 120 traps based on the gross margin SERF analysis. However, the SERF 
results showed that large-sized traps would be the choice of a risk-neutral DM. Alternatively, small-sized traps would be the choice of a risk-averse DM. This result indicates the importance of risk taking for a business to be successful, i.e., no profitable business exists without taking a certain level of risk.

Figure 7. Stochastic efficiency with respect to a function (SERF) for the size of traps (negative exponential utility function).

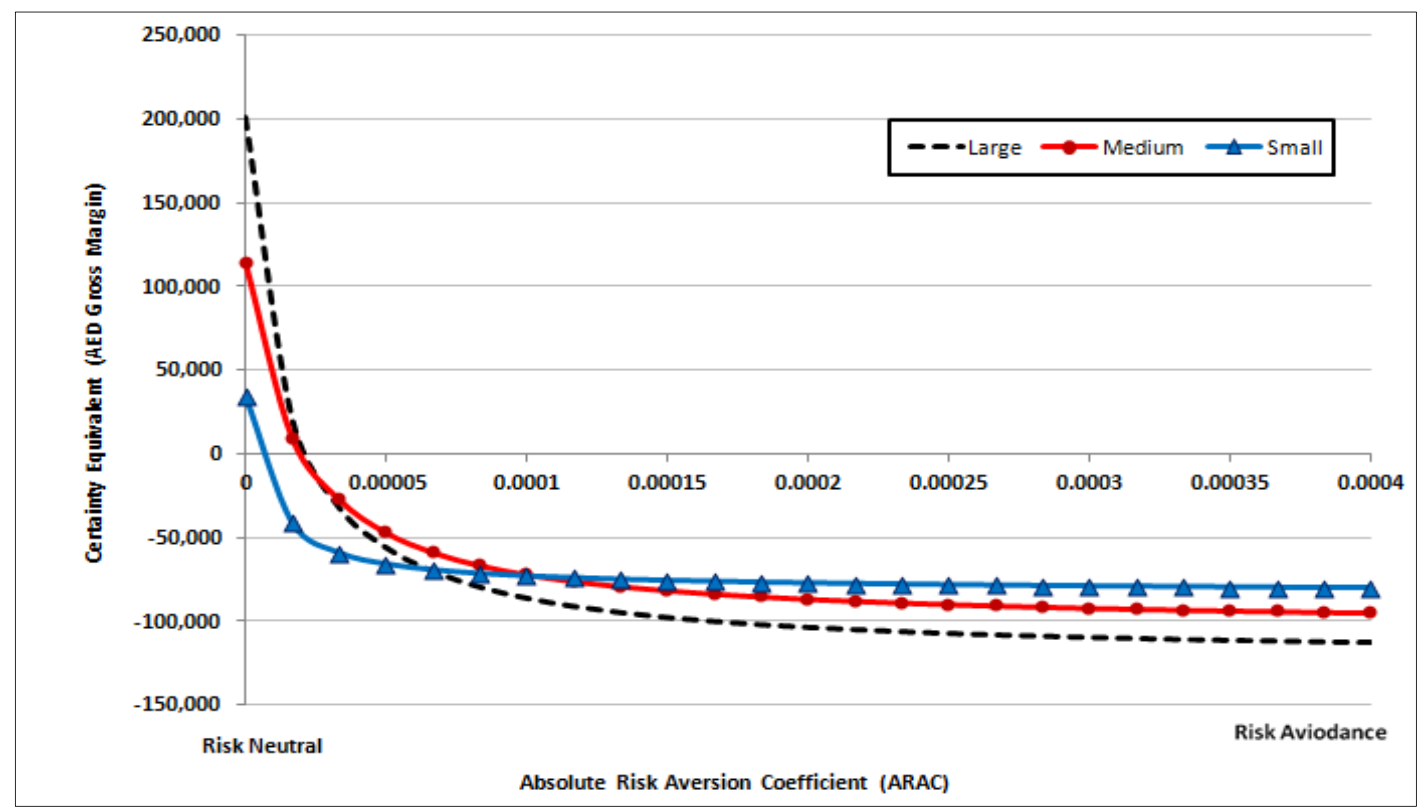

Figure 8. Stochastic efficiency with respect to a function (SERF) for the number of traps (negative exponential utility function).

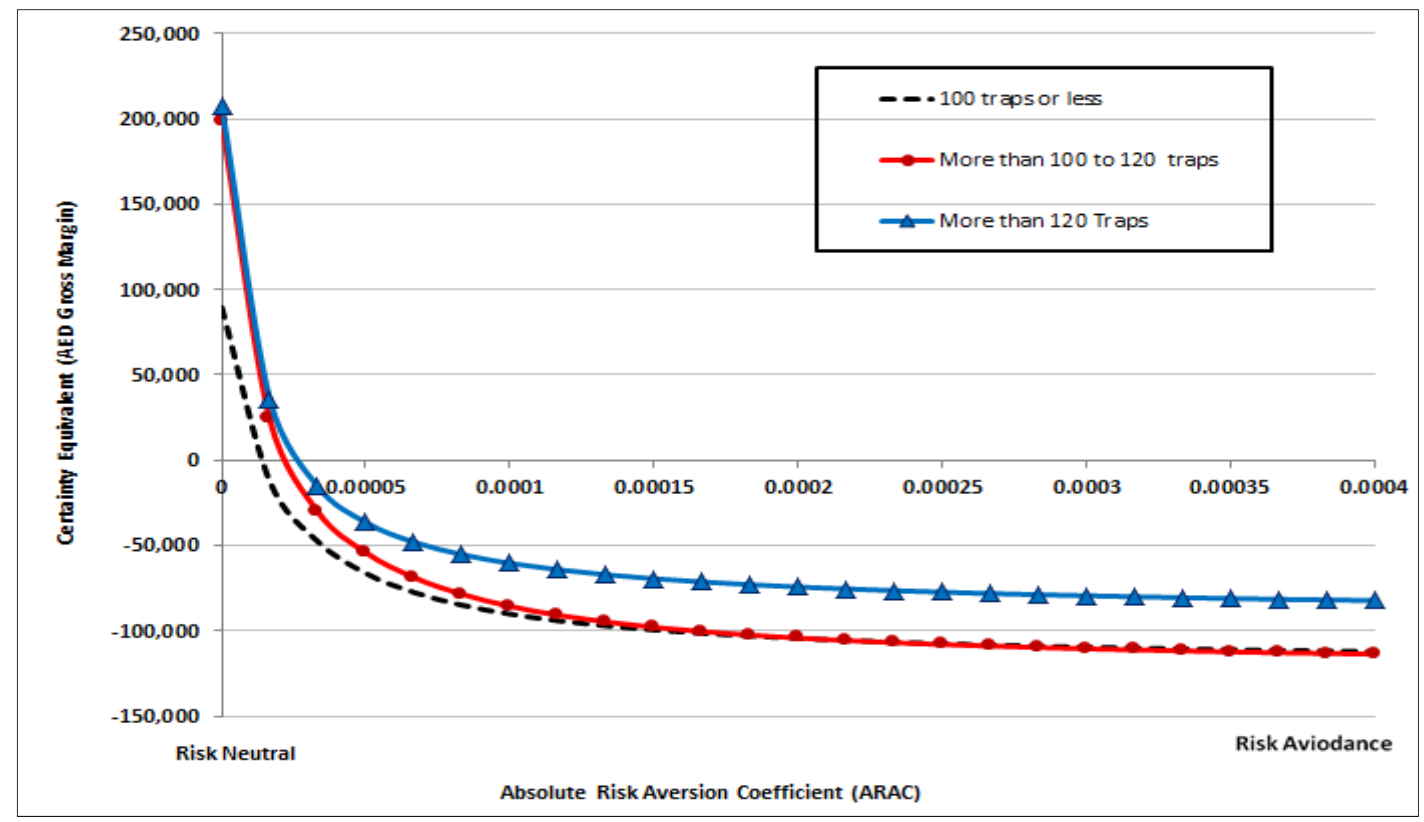

The Emirate of Abu-Dhabi, UAE, fisheries survey of risk and uncertainty analysis showed both agreement and disagreement based on the analysis of the fishing management alternatives. All stochastic dominance risk analysis methods showed that the trap fishing method alternative was preferred over the net and thread alternatives at all levels of attitude towards risk. Furthermore, all 
stochastic dominance risk analysis methods showed that the large number of traps (100 traps or more) alternative was preferred over the smaller and medium number of traps alternatives. However, the SERF analysis results showed that a DM who is risk neutral would prefer large-sized traps over medium- or small-sized traps. This is not the case for a risk-averse DM, who would prefer the small-sized trap alternative over the medium- and large-sized alternatives.

\section{Summary and Conclusions}

The Emirate of Abu-Dhabi, UAE, fishermen operate under extreme risk and uncertainty conditions. The UAE fishery sector has experienced multiple challenges in the last three decades (1980-2010). To analyze the sources of risk that face both fishermen and fisheries in the Emirate of Abu-Dhabi, UAE, two major risk analysis methods were considered: standard stochastic dominance techniques (first/second degree stochastic dominance and stochastic dominance with respect to a function) and stochastic efficiency with respect to a function (SERF). Stochastic dominance techniques and SERF allow the DM to rank risky fishing management alternatives (i.e., fishing methods, size of traps and number of traps). The risk analysis (based on survey information obtained from 131 Emirate of Abu-Dhabi, UAE, fishermen) showed that the coefficients of variation (CV) for the annual gross margin and the monthly number of fishing trips were estimated at 1.25 and 0.61 , respectively. First degree stochastic dominance (FSD) and second-degree stochastic dominance (SSD) were utilized to display gross margin (AED) CDFs based on the fishing method, trap size and number of traps. Furthermore, stochastic efficiency with respect to a function (SERF) under a negative exponential utility function between fishing methods, trap sizes and trap numbers was implemented and showed that fishermen's efficient management choices were not the same for risk-neutral decision-makers compared to risk-averse decision-makers. SERF results showed that large-sized traps would be choice of a risk-neutral DM; however, small-sized traps would be the choice of a risk-averse DM. The results illustrate the importance of considering both the attitude towards risk and economic inefficiencies in UAE fishery practices management and in designing successful fishery policies, as well as considering decision-making at the fisherman level. The risk and uncertainty analyses carried out in this paper included analysis of DM behavior using a continuum of stochastic methods across a spectrum of risk preference from risk neutral (i.e., willing to consider some risk) to risk averse. The results show that fishermen who own small-sized traps face more risk compared to fishermen who own large- and medium-sized traps. The results also showed that DM-selected management alternatives based on the size of traps and the number of traps vary from those selected by a risk-neutral DM compared to those selected by a risk-averse DM. The stochastic dominance analysis showed that traps as a management alternative were preferred over both nets and threads for all techniques. Furthermore, results indicate that policy-makers should target small- and medium-sized fishery operations (those that typically own small-sizes traps and/or a small number of traps) to provide technical and business efficiency support and to also increase their ability to manage risk. Finally, the findings are among the first in the literature utilizing stochastic dominance techniques to identify and rank risky fishing management alternatives and also indicate that the adoption of less risky management alternatives by fishermen may augment the sustainability of this important food sector and ensure a sufficient long-term supply of fish in the UAE. 


\section{Author Contribution}

All the authors contributed equally to this work.

\section{Conflicts of Interest}

The authors declare no conflict of interest.

\section{References}

1. FAO. Information on Fisheries management in the United Arab Emirates. Available online: http://www.fao.org/fi/oldsite/FCP/en/ARE/body.html (accessed on 1 June 2013).

2. Ministry of Environment and Water (MOEW). Exploitation, protection and development of the living aquatic resources in the waters of the state of the United Arab Emirates. Available online: http://www.moew.gov.ae/Portal/en/laws-and-legislations/federal-laws.aspx\#page=1 (accessed on 15 June 2013).

3. Arab Organization for Agricultural Development (AOAD). Arab Agricultural Statistical Database. Available online: http://www.aoad.org/publications.htm (accessed on 25 June 2013).

4. Ministry of Environment and Water (MOEW). Stop the new registration of speedy fishing boats. Available online: http://www.moew.gov.ae/Portal/en/laws-and-legislations/executive-by-laws.aspx (accessed on 15 June 2013).

5. Abu-Dhabi Environment Agency. Fishers Statisical Bulletin. Available online: https://www.ead.ae/en/portal/fisheries.statistical.bulletin.aspx (accessed on 15 July 2013).

6. Kirkley, J.; Paul, C.J.M.; Squires, D. Deterministic and stochastic capacity estimation for fishery capacity reduction. Mar. Resour. Econ. 2004, 19, 271-294.

7. Sharma, K.R.; Leung, P. Technical efficiency of the longline fishery in Hawaii: An application of a stochastic production frontier. Mar. Resour. Econ. 1999, 13, 259-274.

8. Pascoe, S.; Coglan, L. The contribution of unmeasurable inputs to fisheries production: An analysis of technical efficiency of fishing vessels in the English Channel. Am. J. Agr. Econ. 2002, 84, 585-597.

9. Sherif, S.A.; Gheblawi, M.S. Revenue determinants for Abu-Dhabi fishermen and assessment of input allocative efficiency. Agr. Mar. Sci. 2009, 14, 17-25.

10. Aisyah, N.; Arumugam, N.; Hussein, M.A.; Latiff, I. Factors affecting the technical efficiency level of inshore fisheries in Kuala Terengganu; Malaysia. Int. J. Agr. Manage. Dev. 2012, 2, 49-56.

11. Ahmed, N.; Zander, K.K.; Garnett, S.T. Socioeconomic aspects of rice-fish farming in Bangladesh: Opportunities, challenges and production efficiency. Aust. J. Agr. Resour. Econ. 2011, 55, 199-219.

12. Espino, D.C.; Del Hoyo, J.J.G.; Sharp, B.M.H. Capacity and Capacity Utilization of the "Voracera" Fleet in the Strait of Gibraltar. Mar. Resour. Econ. 2005, 20, 367-384.

13. Singh, R.; Weninger, Q.; Doyle, M. Fisheries management with stock growth uncertainty and costly capital adjustment. J. Environ. Econ. Manage. 2006, 52, 582-599.

14. Herrero, I.; Pascoe, S. Value versus volume in the catch of the Spanish South-Atlantic Trawl Fishery. J. Agr. Econ. 2003, 54, 325-341. 
15. Danielsson, A. Efficiency of catch and effort quotas in the presence of risk. J. Environ. Econ. Manage. 2002, 43, 20-33.

16. Bastardie, F.; Nielsen, J.R.; Andersen, B.S.; Eigaard, O.R. Effects of fishing effort allocation scenarios on energy efficiency and profitability: An individual-based model applied to Danish fisheries. Fish. Res. 2010, 106, 501-516.

17. Maravelias, C.D.; Tsitsika, E.V. Economic efficiency analysis and fleet capacity assessment in Mediterranean fisheries. Fish. Res. 2008, 93, 5-91.

18. Andersen, J.L. The inclusion of stocks in multi-species fisheries: The case of Danish Seiners. Mar. Resour. Econ. 2005, 20, 163-184.

19. Tingley, D.; Pascoe, S.; Coglan, L. Factors affecting technical efficiency in fisheries: Stochastic production frontier versus data envelopment analysis approaches. Fish. Res. 2005, 73, 363-376.

20. Del Hoyo, J.J.G.; Espino, D.C.; Toribio, R.J. Determination of technical efficiency of fisheries by stochastic frontier models: A case on the Gulf of Cadiz (Spain). ICES J. Mar. Sci. 2004, 61, 416-421.

21. Greenville, J.; Hartmann, J.; Macaulay, T.G. Technical efficiency in input-controlled fisheries: The NSW Ocean Prawn Trawl Fishery. Mar. Resour. Econ. 2006, 21, 159-179.

22. Robison, L.J.; Barry, P. The Competitive Firm's Response to Risk; Macmillan Publishing Co.: New York, NY, USA, 1987.

23. Boggess, W.G.; Ritchie, J.T. Economic and risk analysis of irrigation decisions in humid regions. J. Prod. Agric. 1988, 1, 116-122.

24. Williams, J.R. A stochastic dominance analysis of tillage and crop insurance practices in a semiarid region. Am. J. Agr. Econ. 1988, 70, 112-120.

25. Williams, J.R.; Llewelyn, R.V.; Mikesell, C.L. An economic risk analysis of conservation tillage systems for wheat, grain sorghum, and soybeans in the Great Plains. J. Soil Wat. Cons. 1989, 34, 234-239.

26. Greene, W.H. Econometric Analysis, 5th ed.; Prentice-Hall International Inc.: New York, NY, USA, 2003.

27. Weersink, A.; Walker, M.; Swanton, C.; Shaw, J.E. Economic comparison of alternative tillage systems under risk. Can. J. Agr. Econ. 1992, 40, 199-217.

28. Hardaker, J.B.; Richardson, J.W.; Lien, G.; Schumann, K.D. Stochastic efficiency analysis with risk aversion bounds: A simplified approach. Aust. J. Agr. Resour. Econ. 2004, 48, 253-270.

29. Meyer, J.; Richardson, W.; Schumann, K.D. Stochastic efficiency analysis with risk aversion bounds: A correction. Aust. J. Agr. Resour. Econ. 2009, 53, 521-525.

30. Hardaker, J.B.; Huirne, R.B.M.; Anderson, J.R.; Lien, G. Coping with Risk in Agriculture, 2nd ed.; CABI Publishing: Cambridge, MA, USA, 2004.

31. Cochran, M.J. Stochastic dominance: The state of the art in agricultural economics. In Proceedings of Southern Region Project S-180 Seminar: An Economic Analysis of Risk Management Strategies for Agricultural Production Firms, Tampa, FL, USA, 23-26 March 1986.

32 King, P.K.; Robison, L.J. An interval approach to measuring decision maker preferences. Am. J. Agric. Econ. 1981, 63, 510-520.

33. Keeney, R.L.; Raiffa, H. Decisions with Multiple Objectives: Preferences and Value Tradeoffs; Wiley Publishers: New York, NY, USA, 1976. 
34. Lien, G.; Stordal, S.; Hardaker, J.B.; Asheim, L.J. Risk aversion and optimal forest replanting: A stochastic efficiency study. Eur. J. Oper. Res. 2007, 181, 1584-1592.

35. Pendell, D.L.; Williams, J.R.; Boyles, S.B.; Rice, C.W.; Nelson, R.G. Soil carbon sequestration strategies with alternative tillage and nitrogen sources under risk. Appl. Econ. Perspect. Pol. 2007, 29, 247-256.

36. Babcock, B.A.; Choi, E.K.; Feinerman, E. Risk and probability premiums for CARA utility functions. J. Agr. Res. Econ. 1993, 18, 17-24.

37. Richardson, J.W.; Schumann, K.D.; Feldman, P.A. Simulation \& Econometrics to Analyze Risk: Simetar(C) Inc. User Manual; Texas A \& M University: College Station, TX, USA, 2008.

38. United Arab Emirates Ministry of Water and Environment (MOEW). Fisheries Statistics. Available online: http://www.moew.gov.ae/en/knowledge-and-statistics/statistics.aspx\#page=1 (accessed on 12 May 2014).

(C) 2014 by the authors; licensee MDPI, Basel, Switzerland. This article is an open access article distributed under the terms and conditions of the Creative Commons Attribution license (http://creativecommons.org/licenses/by/3.0/). 